\title{
Torsade-de-pointes syndrome with short coupling interval
}

INSERM

\section{Source}

INSERM. (1999). Orphanet: an online rare disease and orphan drug data base. Torsadede-pointes syndrome with short coupling interval. ORPHA:51084

Torsade-de-pointes (T dP) syndrome with short coupling interval is a very rare variant of Torsade de pointes, a polymorphic ventricular tachycardia, which is characterized by a short coupling interval of the first TdP beat on electrocardiogram in the absence of any structural heart disease. It manifests in early adulthood with syncope, often results in ventricular fibrillation and shows a high risk of sudden cardiac death. 